\title{
Bioinformatic Analysis of MIRU-VNTR Repeats of Mycobacterium Tuberculosis Strains from Lagos, Nigeria
}

\author{
Seydou Golo BARRO ${ }^{\mathrm{a}, \mathrm{b}, 11}$, Faouziatou BANCE ${ }^{\mathrm{a}}$, Amadou DICKO $^{\mathrm{a}, \mathrm{c}}$, Michel \\ GOMGNIBOU ${ }^{\mathrm{a}}$ and Pascal STACCINI ${ }^{\mathrm{b}}$ \\ ${ }^{a}$ Nazi Boni University, Bobo-Dioulasso, Burkina Faso \\ ${ }^{b}$ RETINES Lab - IRIS department - Medical Faculty of Nice University, France \\ ${ }^{c}$ National Institute of Public Health, Burkina Faso
}

\begin{abstract}
The analysis of Mycobacterial Interpersed Repetitive Unit- Variable Number of Tandem Repeat (MIRU-VNTR) discriminates against the species of M. tuberculosis involved in the transmission of the disease. The reference method is the manual method. Our study involved developing a bioinformatics method of interpreting MIRU-VNTR and comparing it to the manual method. For this we used two softwares, namely imagej and Microsoft Excel. Imagej was used to determine the migration distance of the bands and for the measure of size in a base pair. The number of repetitions of 18 markers used was analyzed with Excel macro. The results obtained were: $27 \%$ of the results exactly consistent, $16 \%$ of outliers generated by the macro and $57 \%$ of the results not matching.
\end{abstract}

Keywords. tuberculosis, MIRU-VNTR, bioinformatics, imagej software, Excel

\section{Introduction}

Tuberculosis was responsible for 1.451 million deaths in 2018 with a high incidence in Southeast Asia and Africa [1]. It eradication requires the inclusion of molecular biology techniques in the study of mycobacteria.

In the genome of Mycobacterium tuberculosis, there are sequences repeated in tandem, Mycobacterial Interpersed Repetitive Unit- Variable Number of Tandem Repeat (MIRU-VNTR) [2], which help during an epidemiological study. Determining the number of repetitions is a complex analysis that is done manually, method long and tedious, and errors can occur [3]. The present study is part of the implementation of a bioinformatics analysis method for the analysis of MIRU-VNTR. The main objective of this study is to develop a bioinformatics tool for MIRU-VNTR interpretation.

\footnotetext{
${ }^{1}$ Corresponding Author, Golo Seydou Barro, Nazi Boni University, Bobo-Dioulasso, Burkina Faso; Email: golo_seydou@yahoo.fr.
} 


\section{Methods}

Measures on PCR products gel photos were taken with imagej. These data were recorded in Excel where a programmed macro calculate the number of repetitions in order to determine the number of repetitions of each marker. This method were compared to manual one.

\section{Results}

Out of the 252 analyzes, we obtained 73 outrageous results, include 40 results corresponding to samples without bands and 33 results corresponding to samples that have 0 replicates; and 179 correct results which include 100 results that do not agree with manual method results and 67 exactly, obtained with the two methods. In view of the results, the concordance rate is $31.60 \%$, i.e. 67 out of 212 analyzes.

\section{Discussion}

The results obtained with the macro gave us 67 results which correspond to the results obtained with the manual method so a match rate of $27 \%$. The results that do not match, contain results that do not appear on the gel, and those that gave too high values. These results represent $16 \%$ of the total. Ignoring these results, the results of bioinformatics and manual analyzes have a concordance rate of $31.60 \%$. The difference in the results obtained with the two methods could be explained by the way of determining the migration distance and the photos quality.

\section{Conclusion}

The aim of this study was to develop a bioinformatics method for interpreting MIRUVNTRs, Imagej and Excel software were used. The results obtained with the Excel macro were compared with the results of the manual method. These results agree at $31.60 \%$ and are encouraging. This method must be optimized to be validated.

\section{References}

[1] WHO. World Tuberculosis Report 2018; 2018.

[2] TAZI L. Analysis of genetic diversity and population structure of Mycobacterium tuberculosis for the study of tuberculosis transmission in Casablanca, Morocco [Doctoral thesis]. (2002, December).

[3] Sahraoui N, Muller B, Djamel Y, Fadéla B, Rachid O, Jakob Z, Djamel G. Evaluation of VNTR (variable number of tandem repeats) of Mycobacterium bovis isolates in Algeria. Annales de biologie clinique. 2010;68(4):449453. 